\title{
Maser Source-Finding Methods in HOPS
}

\author{
A. J. Walsh ${ }^{\mathrm{A}, \mathrm{F}}$, C. Purcell ${ }^{\mathrm{B}}$, S. Longmore ${ }^{\mathrm{C}}$, \\ C. H. Jordan ${ }^{\mathrm{A}, \mathrm{D}}$, and V. Lowe $\mathrm{D}, \mathrm{E}$

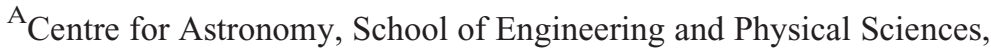 \\ James Cook University, Townsville, QLD 4814, Australia \\ ${ }^{\mathrm{B}}$ School of Physics and Astronomy, University of Leeds, Leeds, LS2 9JT, UK \\ ${ }^{\mathrm{C}}$ European Southern Observatory, Karl-Schwarzschild-Str. 2, 85748 Garching, Germany \\ ${ }^{\mathrm{D}}$ CSIRO Astronomy and Space Science, PO BOX 76, Epping, NSW 1710, Australia \\ ${ }_{\text {Echool of Physics, University of NSW, Sydney, NSW 2052, Australia }}$ \\ ${ }^{\mathrm{F}}$ Corresponding author. Email: andrew.walsh@jcu.edu.au
}

\begin{abstract}
The $\mathbf{H}_{2} \mathbf{O}$ Southern Galactic Plane Survey (HOPS) has observed $100 \mathrm{deg}^{2}$ of the Galactic plane, using the Mopra radio telescope to search for emission from multiple spectral lines in the 12-mm band $(19.5-27.5 \mathrm{GHz})$. Perhaps the most important of these spectral lines is the 22.2-GHz water-maser transition. We describe the methods used to identify water-maser candidates and subsequent confirmation of the sources. Our methods involve a simple determination of likely candidates by searching peak emission maps, utilising the intrinsic nature of water-maser emission, spatially unresolved and spectrally narrow-lined. We estimate completeness limits and compare our method with results from the DUCHAMP source finder. We find that the two methods perform similarly. We conclude that the similarity in performance is due to the intrinsic limitation of the noise characteristics of the data. The advantages of our method are that it is slightly more efficient in eliminating spurious detections and is simple to implement. The disadvantage is that it is a manual method of finding sources and so is not practical on datasets much larger than HOPS, or for datasets with extended emission that needs to be characterised. We outline a two-stage method for the most efficient means of finding masers, using DUCHAMP.
\end{abstract}

Keywords: masers — stars: formation — surveys — techniques: spectroscopic

Received 2011 October 21, accepted 2011 November 24, published online 2011 December 16

\section{Introduction}

Astrophysical masers have been known for over forty years (Weaver et al. 1965; Gundermann 1965). They are known to occur in transitions of many chemical species, with the most common seen in hydroxyl $(\mathrm{OH})$, methanol $\left(\mathrm{CH}_{3} \mathrm{OH}\right)$, silicon monoxide $(\mathrm{SiO})$ and water $\left(\mathrm{H}_{2} \mathrm{O}\right)$. The 22.2- $\mathrm{GHz}_{2} \mathrm{O}$-maser transition is the strongest and most widespread known to date. It is seen towards a variety of astrophysical objects such as low- and high-mass star forming regions (e.g. Cheung, Rank \& Townes 1969; Forster \& Caswell 1999; Claussen et al. 1996) evolved stars (Dickinson 1976; Miranda et al. 2001; Hinkle \& Barnes 1979; Barlow et al. 1996) and the centres of active galaxies (Claussen et al. 1984). Other masers are often seen arising from the same sites that give rise to $\mathrm{H}_{2} \mathrm{O}$ masers. In particular, $\mathrm{OH}$ and $\mathrm{CH}_{3} \mathrm{OH}$ masers occur within the spatial resolution of observations (typically an arcsecond or less) of $\mathrm{H}_{2} \mathrm{O}$ masers in high-mass star forming regions (Forster \& Caswell 2000; Walsh et al. 1998). This has led to the identification of a tentative evolutionary sequence where both $\mathrm{CH}_{3} \mathrm{OH}$ and $\mathrm{H}_{2} \mathrm{O}$ masers appear early on during the formation process, with $\mathrm{OH}$ masers appearing at a more evolved stage, but with some overlap between all three maser species. Such arguments are based on the statistical occurrence of the masers towards other signposts of high-mass star formation, such as ultracompact Hir regions and hot cores. However, to date most searches for these masers have targeted known regions of star formation and thus may suffer from biases. In order to reduce such biases of candidate selection, it is important to conduct untargeted surveys of the Galaxy for these masers.

The methanol multibeam project (Caswell et al. 2010) is one such survey, which focuses on the strongest and most widespread of the Class II $\mathrm{CH}_{3} \mathrm{OH}$ masers ${ }^{1}$ at 6.7 GHz. This survey is designed to cover the entire Galactic plane in a 4-deg wide latitude band. In the near future, there will be a survey on the Australian SKA Pathfinder radio telescope (ASKAP), called GASKAP, which will survey the southern Galaxy up to 10 deg away from the Galactic plane. One of the target spectral species

\footnotetext{
${ }^{1}$ Class I $\mathrm{CH}_{3} \mathrm{OH}$ masers are collisionally pumped and are usually associated with weak shocks whereas Class II $\mathrm{CH}_{3} \mathrm{OH}$ masers are radiatively pumped and closely associated with the early stages of high mass star formation (e.g. Voronkov et al. 2005 and references therein).
} 
for GASKAP is $\mathrm{OH}$, including the main maser lines at 1.665 and $1.667 \mathrm{GHz}$. The $\mathrm{H}_{2} \mathrm{O}$-maser line has recently been surveyed as part of the $\mathbf{H}_{\mathbf{2}} \mathbf{O}$ southern Galactic Plane Survey (HOPS), using the Mopra radiot elescope (Walsh et al. 2011). HOPS has observed $100 \mathrm{deg}^{2}$ of the Southern Galactic Plane at $12 \mathrm{~mm}$ and includes emission from the 22.2- $\mathrm{GHz} \mathrm{H}_{2} \mathrm{O}$ maser as one of its main target lines. HOPS forms the basis for the work reported in this paper.

With the advent of these untargeted, large scale surveys, the problem of finding and identifying new sources has become an important challenge. HOPS is the smallest of the surveys mentioned above, in terms of equivalent beam pointings in the survey area, with approximately 90000 individual beams. However, the Mopra spectrometer delivers 4096 channels for each of 16 simultaneous spectra yielding a dataset with nearly $6 \times 10^{9}$ independant voxels ( $l-b-v$ data elements). This number is prohibitively large such that searching each individual spectrum for maser emission by eye becomes impractical.

In this paper, we describe the source finding method used in HOPS to identify $\mathrm{H}_{2} \mathrm{O}$ masers and compare its performance to the new source-finding program, DUCHAMP.

\section{HOPS Data}

Details of how the Mopra observations were undertaken and the resultant data have been described elsewhere (Walsh et al. 2011). However, we summarise the relevant details here for convenience. Data were accumulated in square tiles that are $0.5 \mathrm{deg}$ on each side. The tiles were oriented in Galactic coordinates and positioned either at $b=+0.25^{\circ}$ or $b=-0.25^{\circ}$. Each tile was observed twice, where one observation was scanned in Galactic longitude and the other in Galactic latitude. This greatly reduces (but does not completely eliminate) scanning artifacts that appear as strips in the data cubes. Observing each tile twice also reduces the noise. The resulting data were regridded onto data cubes with $30 \times 30^{\prime \prime}$ pixels. The Mopra beam at $22.2 \mathrm{GHz}$ is approximately $2.2^{\prime}$. The final data cubes were typically strips 10-deg long and 1-deg wide. The spectral channel width in the data cubes is $0.45 \mathrm{~km} \mathrm{~s}^{-1}$. The main $\mathrm{H}_{2} \mathrm{O}$-maser data cube covers a velocity range of approximately -570 to $+1290 \mathrm{~km} \mathrm{~s}^{-1}$. Each $\mathrm{H}_{2} \mathrm{O}$-maser cube consists of $1203 \times 125 \times 2203$ pixels and is $1.3 \mathrm{~GB}$ in size. Since observations were taken under varying weather conditions and telescope elevations, the noise level varies by a factor close to 2 .

\section{Source-Finding Methods}

Here we outline two source-finding methods that are generally available and their applicability to $\mathrm{H}_{2} \mathrm{O}$-maser finding in HOPS data. We also describe HOPSfind, which is the method we used to detect $\mathrm{H}_{2} \mathrm{O}$ masers in HOPS.

\subsection{CLUMPFIND}

CLUMPFIND (Williams, de Geus \& Blitz 1994) is an automated source-finding method that was designed to characterise spectral line emission from molecular clouds. Not only does it find regions of emission, but it also attempts to break up overlapping regions of emission into individual detections. For observations that show complex, extended emission, it is very useful in characterising detections by breaking up the emission along boundaries in an intuitive fashion (e.g. Walsh et al. 2007). However, the program is computationally intensive, with most of the computational power spent on identifying boundaries of extended emission and separating possibly overlapping detections. The $\mathrm{H}_{2} \mathrm{O}$-maser data from HOPS show very little overlap between individual maser sites. Also, we do not expect any of the maser sites to be resolved in the Mopra beam. Thus, we regard CLUMPFIND as too inefficient for our purposes.

\subsection{DUCHAMP}

DUCHAMP $^{2}$ (Whiting 2011) is a source-finding program designed with large surveys, particularly those associated with ASKAP, in mind (Johnston et al. 2007). It does not require emission to conform to a particular shape (e.g. Gaussian) or size, other than the user-defined inputs. It works by searching through data cubes for emission above a certain cutoff level and then merging detections, based on user-defined inputs. The user-defined inputs that are relevant to this work are the region within the cube to search, a threshold (expressed in terms of $\sigma$ ) over which voxels will be counted towards a detection, the minimum number of pixels to constitute a detection, the minimum number of channels which constitute a detection and how far away voxels are allowed to be from each other in order to be considered in a merged detection.

DUCHAMP also offers the option to reconstruct the data cube using the à trous procedure. This procedure allows the reliable detection of fainter sources and the removal of spurious detections in a noisy data cube by effectively removing some of the random noise. More details of this method can be found on the DUCHAMP website. ${ }^{2}$

\subsection{HOPSfind}

The limitation of any source-finding method is the amount of time available to complete the task, so the choice of source-finding method is one of efficiency: how to identify the largest number of real sources without identifying too many spurious sources in a reasonable time-frame. During the HOPS pilot observations and data processing (Walsh et al. 2008) it was realised that each square degree of the Galactic plane is expected to have no more than about $10 \mathrm{H}_{2} \mathrm{O}$ masers, meaning that overlapping masers would be rare. Also, because the masers are typically spatially unresolved, bright (many Janskys) and narrow-lined (less than $5 \mathrm{~km} \mathrm{~s}^{-1}$ ), it is relatively easy to manually identify them, compared to weaker, extended emission. Therefore, we used the following method

\footnotetext{
${ }^{2}$ Version 1.1.13, http: / / www.atnf.csiro.au/computing/ software/duchamp
} 
(which we call 'HOPSfind') to identify $\mathrm{H}_{2} \mathrm{O}$ masers in HOPS.

Each processed data cube was smoothed with a twodimensional Gaussian kernel of size $90 \times 90^{\prime \prime}$. From each data cube, a peak temperature map (PTM) was made. The PTM is made, using the MIRIAD task MOMENT and choosing the 'mom $=-2$ ' option. This option scans through the spectrum at each spatial pixel and finds the brightest emission, which is then copied to the PTM at the same position. Thus, the PTM is similar in appearance to the more commonly used ' 0 th moment', or integrated intensity map (IIM). However, the PTM has one significant advantage over an IIM. The HOPS IIM tends to be dominated by small errors in the baseline, if it is not completely flat. This is because we have a broad band (137.5 MHz across 4096 channels), where the small baseline errors accumulate in the IIM.

Until recently, radio-telescope observations have been made with narrow bandwidths and with few channels, where small errors do not dominate in an IIM. It is with the advent of much broader bandwidths and many thousands of channels that the IIM becomes less reliable. We also note that attempts to remove the baseline are difficult because the baseline is usually complicated over the full extent of the band. A low-order polynomial may be removed from the baseline, improving the result, but small-scale deviations still tend to be significant in an IIM. Because this problem is likely to affect many spectral line surveys in the future, we suggest that PTMs are used in favour of IIMs for locating emission. Figure 1 shows a comparison of an IIM (top) and PTM (bottom). In the IIM, it is possible to clearly identify one maser, but many more are seen in the PTM.

We found that the PTMs are very useful in identifying most of the masers in the survey. However, the $\mathrm{H}_{2} \mathrm{O}$ maser spots appear as peaks that are typically broader than the spectral channel width $\left(0.45 \mathrm{~km} \mathrm{~s}^{-1}\right)$, so our method can be improved by averaging adjacent channels together before producing the PTM. Since we found that the average maser line width is $1.4 \mathrm{~km} \mathrm{~s}^{-1}$, we average $3 \times 0.45 \mathrm{~km} \mathrm{~s}^{-1}$ channels together.

Maser candidates were then visually checked, using the PTMs and by inspection of the spectrum and raw data cube at maser candidate positions. A maser candidate was deemed confirmed if the peak signal was greater than five-times the noise level $(5 \sigma)$ in at least two adjacent channels or if there were at least three adjacent channels that were $3 \sigma$ or greater in the raw (i.e. unaveraged) data. We also checked for any signals appearing in a single channel above $8 \sigma$, but found no new masers that did not already satisfy the above requirements. Using this method, we identified 381 masers (approximately 71\% of all masers identified).

$\mathrm{H}_{2} \mathrm{O}$ masers often occur with multiple peaks in the spectrum. Each of these peaks (called maser spots) are typically found spatially close to each other, on scales of a few arcseconds or less. The grouping of maser spots is referred to as a maser site. The spots within each site are
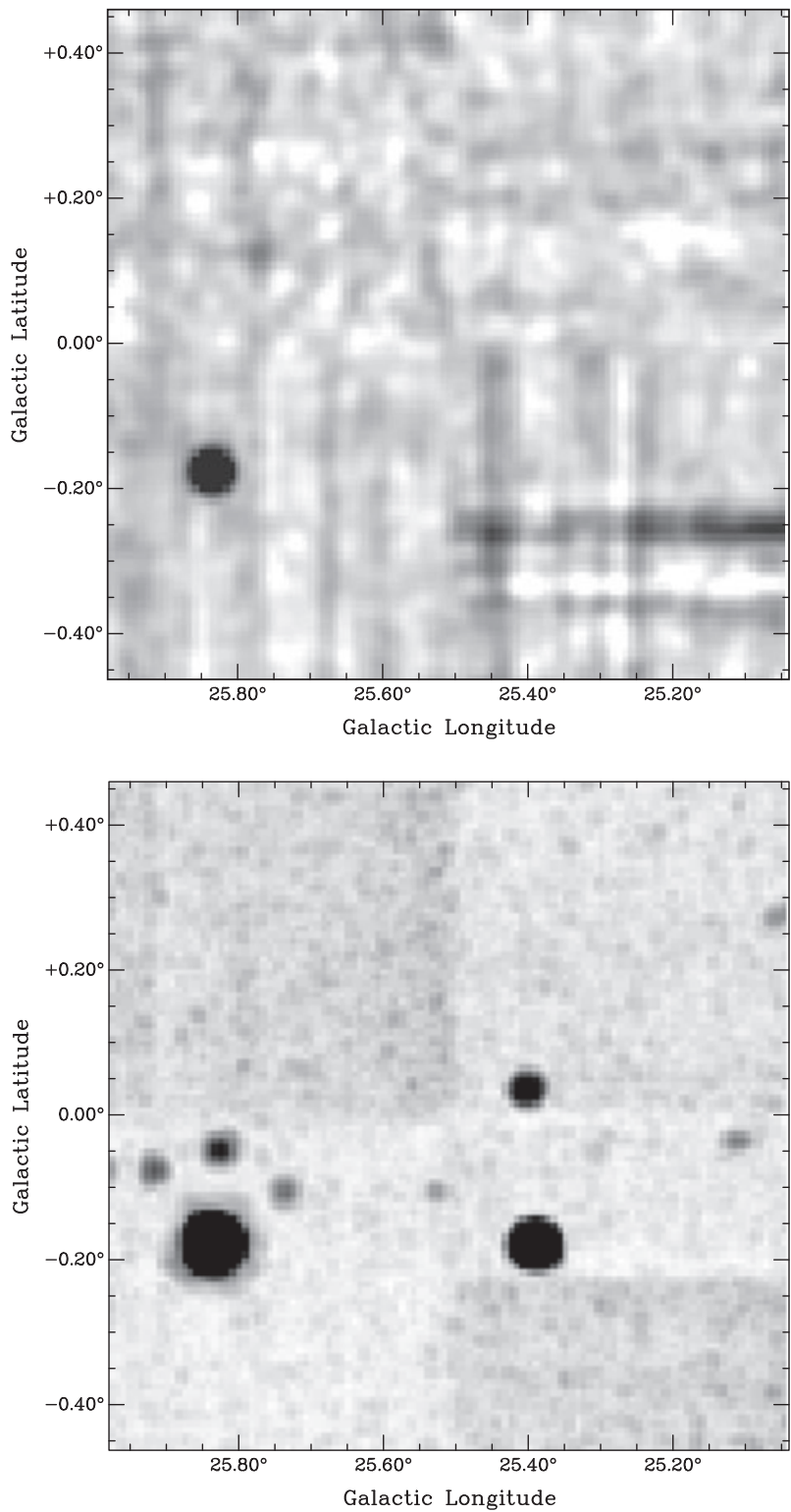

Figure 1 Examples of moment maps used to identify $\mathrm{H}_{2} \mathrm{O}$ masers in HOPS. (Top): Integrated intensity, or 0th moment map, where only one maser can be clearly seen at $l=25.83, b=-0.18$. (Bottom): Peak temperature, or -2 moment map, showing 10 masers.

expected to be associated with a single site of star formation. Multiple instances of maser sites are also often found within the same star forming cloud. Given the Mopra beam, it is not possible to resolve a single maser site or multiple maser sites that are within the Mopra beam. Thus, if we see multiple peaks within a spectrum at a single location, we only assign it to a single detection, even though it is possible that many single detections are comprised of multiple maser sites.

Any weak maser candidates that were not confirmed in the initial list of 381 masers were reobserved with Mopra, using two minutes on-source plus two minutes off-source integration time, resulting in a noise level about $\sqrt{2}$ lower than the initial observations. Any spectral features that 
Table 1. Input parameters for DUCHAMP

\begin{tabular}{lcl}
\hline Parameter & Value & Description \\
\hline minPix & 15 & Must have at least 15 spatial pixels in one velocity channel \\
minChannels & 2 & Must include emission in at least two channels \\
minVoxels & 26 & Must have at least 26 pixels in any channel in the cube \\
snrCut & 3 & Pixels must be at least 3× the rms noise level \\
flagAdjacent & true & Channels must be adjacent to be merged \\
\hline
\end{tabular}

were not common in both observations were discarded as spurious. Unfortunately, because $\mathrm{H}_{2} \mathrm{O}$ masers are known to vary, it is possible that some real masers detected in the first observations were below the detection limit of the second observations and thus discarded.

In all, we identified 540 confirmed $\mathrm{H}_{2} \mathrm{O}$ masers, using the two-stage method outlined above.

\section{Source Finding with DUCHAMP}

As described above, DUCHAMP is an automated sourcefinding method that we can utilise to compare HOPSfind for detecting $\mathrm{H}_{2} \mathrm{O}$ masers. DUCHAMP uses a single detection threshold for the entire cube, rather than taking into account local noise levels, as is naturally done by the human eye. Therefore, in order to create the best data cube for DUCHAMP to search for sources, it is necessary to further process the data. We average a section of the $\mathrm{H}_{2} \mathrm{O}$-maser data cube that is free of line emission $\left(500-1000 \mathrm{~km} \mathrm{~s}^{-1}\right)$ and use the resultant 2-dimensional image as an rms noise map. The full $\mathrm{H}_{2} \mathrm{O}$-maser cube is then divided by the noise map to effectively create a signal-to-noise cube, where the majority of noise variations have been smoothed out. It is this signal-to-noise cube that we use DUCHAMP to search for source.

Successful operation of DUCHAMP requires fine tuning of the input parameters so that as many real sources are found, with minimal spurious detections. At some level, there is a trade off between how deep into the noise one chooses to search for weak masers and how much extra time is required to eliminate spurious detections. This applies equally to any source-finding method.

Our most efficient input parameters that we chose for DUCHAMP are given in Table 1. These input parameters effectively mean that there must be emission over at least one beam in a single spectral channel and that a source must comprise of emission in at least two adjacent channels. This effectively eliminates the chance of finding masers that appear in only one spectral channel. However, our experience with HOPSfind found no such masers, so that these requirements become an effective method to remove spurious sources that may appear in a single channel. The requirement that there is emission in at least two adjacent channels is similar to the 3-channel averaging used in HOPSfind. We found that using only two adjacent channels and not three, as well as a cutoff level set at $3 \sigma$, gives results most closely matched to HOPSfind, allowing for a direct comparison.

\section{Comparison of HOPSfind with DUCHAMP}

An important consideration in the choice of any sourcefinding method is how much time is required. We found that to search each square degree for $\mathrm{H}_{2} \mathrm{O}$ masers, HOPSfind typically took about 10 minutes so that the entire survey region can be searched in around 1000 minutes. Most of this time is taken in transcribing the coordinates of detected masers to a source file and checking correctness of the transcribed details. The time taken for DUCHAMP to search each 10-deg strip was approximately 4.5 minutes so the entire $100 \mathrm{deg}^{2}$ of the survey region was searched in approximately 45 minutes. Thus, the running time for DUCHAMP is significantly less than for HOPSfind. However, this time does not take into account the time required to set up each method, which is very short for HOPSfind but typically much longer for DUCHAMP. This is due to time taken to alter input parameters in order to find the most efficient balance when running DUCHAMP. Thus, on small datasets, a manual source-finding method such as HOPSfind is quicker to implement and complete. On much larger datasets, HOPSfind will take much longer due to the time taken to catalogue each detection.

Overall the DUCHAMP source-finding method found 620 detections. Of the 620 detections identified by DUCHAMP, 491 of them overlap with confirmed masers from HOPSfind. The majority of the remainder are considered spurious detections after visually checking the candidates, as was done for HOPSfind. A large fraction of the spurious detections using DUCHAMP appear to be artifacts along the scanning directions used in the observations. Inspection of the raw data cubes in these instances reveals linear features in either Galactic longitude or latitude and spurious detections are often made where two such scanning artifacts meet. Such features are easy for the human eye to identify, but difficult for an automated method. The scanning artifacts are generally of two types: a 'ripple' in the spectral dimension caused by a poorly subtracted baseline, or a region of elevated noise.

Examples of spurious detections are shown in Figures 2 and 3. Figure 2 shows a peak in the spectrum at around $-155.3 \mathrm{~km} \mathrm{~s}^{-1}$, consisting of at least two adjacent channels. These two channels are shown as maps from the data cube in Figure 3. The positions of confirmed $\mathrm{H}_{2} \mathrm{O}$ masers are shown in Figure 3, with plus symbols and the location of detections made by DUCHAMP are outlined with black solid lines. As can be seen from Figure 3, most 


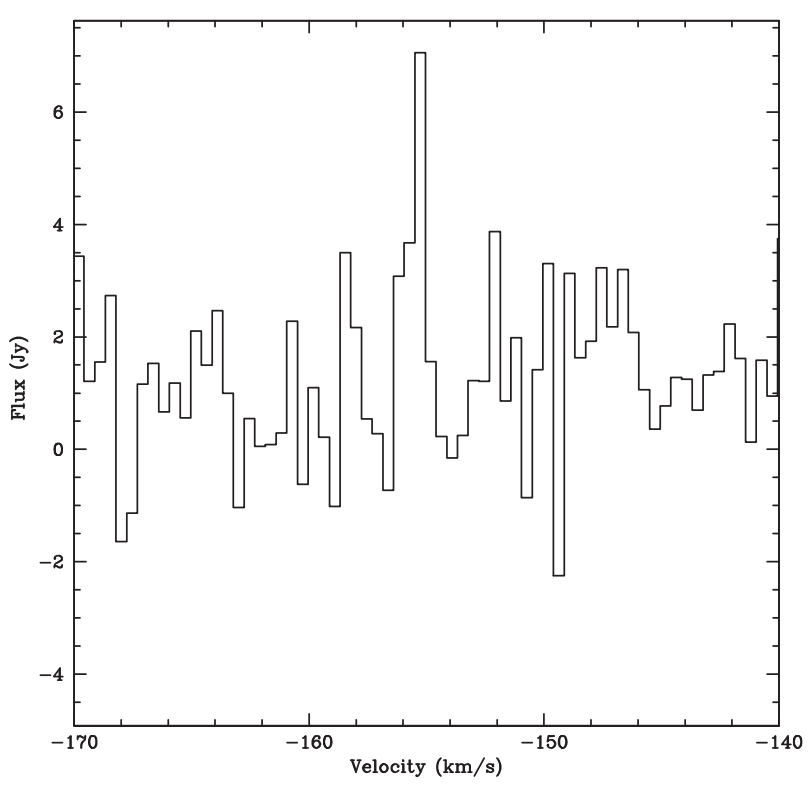

Figure 2 Spectrum of the spurious detection made by DUCHAMP at the position $(l, b)=(25.43,-0.38)$. The strongest peak is found at a velocity of $-155.3 \mathrm{~km} \mathrm{~s}^{-1}$, with an adjacent channel at $-155.7 \mathrm{~km} \mathrm{~s}^{-1}$.

of the DUCHAMP detections coincide with confirmed masers. However, there are three DUCHAMP detections which do not coincide with confirmed $\mathrm{H}_{2} \mathrm{O}$ masers at $(l, b)=(25.42,-0.25),(25.43,-0.38)$ and $(25.28$, $-0.25)$. The DUCHAMP detection represented in Figure 2 is located at $(25.43,-0.38)$. The two channels of the data cube do not show a spatially confined peak of emission at this position. Instead, both channels show positive scanning artifacts that go through this position. The channel at $-155.3 \mathrm{~km} \mathrm{~s}^{-1}$ shows a scanning artifact at a Galactic longitude of $-0.38^{\circ}$ and the channel at $-155.7 \mathrm{~km} \mathrm{~s}^{-1}$ shows a weaker scanning artifact at a Galactic latitude of $25.42^{\circ}$. Thus, DUCHAMP identifies the convergence of these two artifacts as a detection. Inspection of the data cube at the positions and velocities of the other two DUCHAMP detections that do not coincide with a confirmed $\mathrm{H}_{2} \mathrm{O}$ maser indicate that they too are the result of scanning artifacts.

It may be possible in the future to automatically eliminate some of these spurious detections by imposing a limit on the maximum size, or number of pixels that constitute a detection. The maximum size would be set at slightly larger than the beam FWHM. This is because we assume that $\mathrm{H}_{2} \mathrm{O}$-maser detections will always appear unresolved in our observations. Unfortunately, it is not possible to specify a maximum number of pixels in DUCHAMP. Even if this were possible, care must be used in removing such spurious detections, since very strong masers will also be identified as spurious, as emission above the detection threshold will cover a larger area than the beam FWHM. This can be seen in Figure 3 where the DUCHAMP detection associated with the brightest $\mathrm{H}_{2} \mathrm{O}$ maser, located at $(25.83,-0.17)$, includes pixels that cover an area much larger than the beam FWHM.
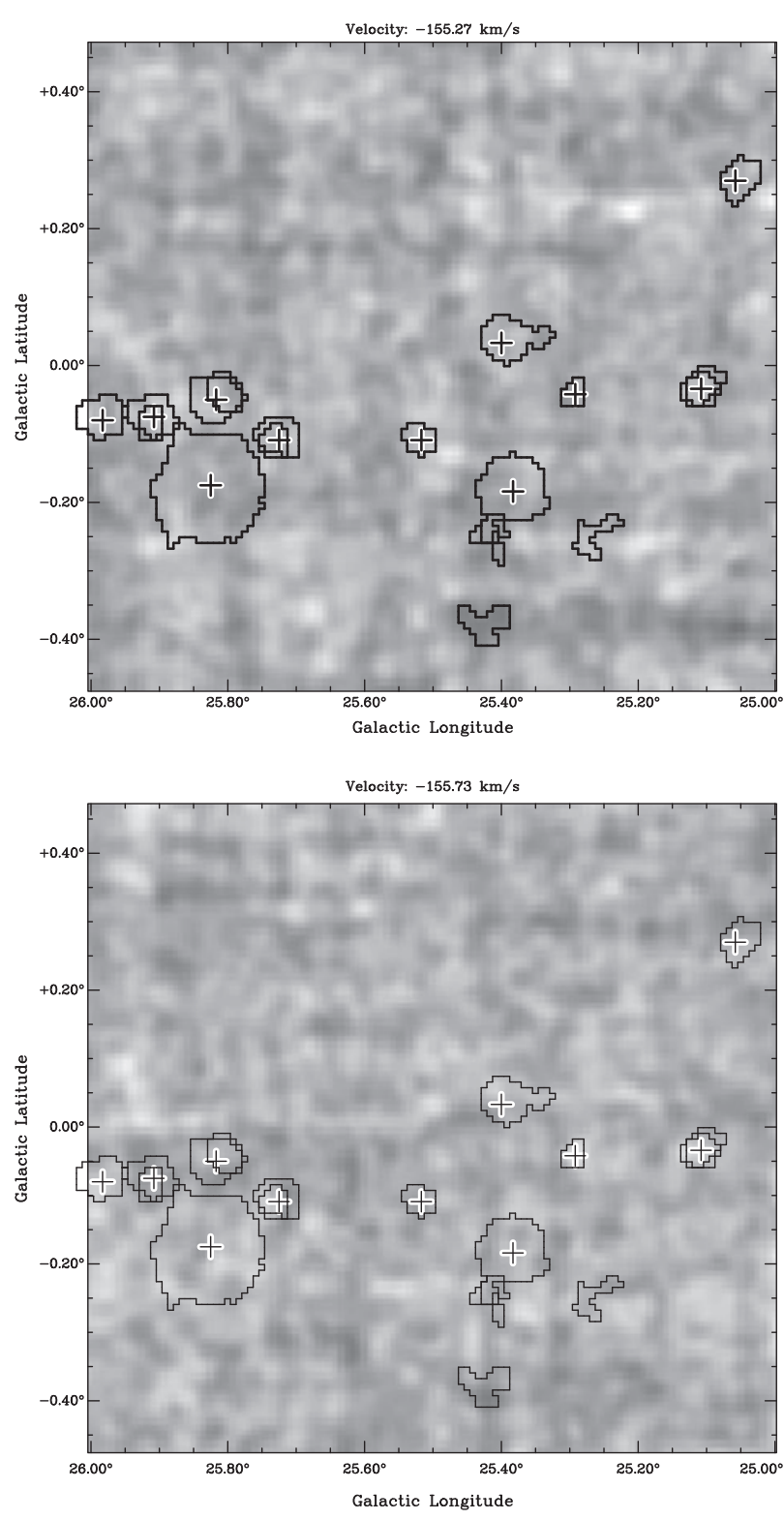

Figure 3 Two adjacent velocity channels are shown in the G025-026 region. The plusses indicate positions of confirmed $\mathrm{H}_{2} \mathrm{O}$ masers and the regions outlined with black lines are the results of DUCHAMP. Within the field of view, there are three DUснамP detections that are not associated with confirmed $\mathrm{H}_{2} \mathrm{O}$ masers and are most likely spurious detections centred on $(l, b)=(25.42$, $-0.25),(25.43,-0.38)$ and $(25.28,-0.25)$.

It is likely that a two-stage approach will be most useful: The first stage identifies strong masers above a high cutoff level, with no limitation on the maximum number of pixels in a detection. The second stage uses a low cutoff level to search for the weakest masers and also uses a maximum number of pixels to eliminate spurious detections much larger than the beam size.

Another approach is to remove scanning artifacts prior to running Duchamp. Purcell et al. (2011) have successfully demonstrated that most baseline ripples may be removed from HOPS data by fitting low-order polynomials to the line-free channels so that the performance 
of DUCHAMP is greatly improved. Scanning artifacts which manifest as regions of elevated noise may be mitigated by dividing each spectral plane by a 'noise-map' to create a signal-to-noise cube, as described above.

Of the 129 detections found by DUCHAMP, but not confirmed as $\mathrm{H}_{2} \mathrm{O}$ masers, we find 11 of them that are possibly real detections, based on their size, shape and spectra, that were not identified using HOPSfind. These maser candidates await followup observations with Mopra to confirm them as real masers, or otherwise.

\subsection{Comparison of Completeness Limits}

An alternate method of comparing our source-finding method to DUCHAMP is to test the efficiency of both methods in detecting weak sources that have been artificially created in a source-free data cube. Note that we used this method in HOPS to determine the completeness limit of HOPSfind (Walsh et al. 2011) and details can be found therein. We used the HOPS data within the $l=296$ to $297 \mathrm{deg}^{2}$ as our template, in which we did not detect any confirmed masers, but has noise characteristics representative of the full survey. Using the MIRIAD task IMGEN, we injected five sources, with typical $\mathrm{H}_{2} \mathrm{O}$-maser characteristics (i.e. FWHM of $1.4 \mathrm{~km} \mathrm{~s}^{-1}$ ), at random positions and velocities into the data cube and searched for detections using HOPSfind and DUCHAMP. This was repeated twenty times so that in total 100 sources were searched for. We varied the intensity of the generated sources to four values, equivalent to a peak of 5.5, 6.7, 8.4 and 11.1 Jy. The rms noise level in the data cube was $1.5 \mathrm{Jy}$. We also performed the source-finding on a signalto-noise cube, as described in Section 5.

Figure 4 shows the success rate of detecting the generated sources in the data cube for HOPSfind and DUCHAMP. We find that the methods have very similar success rates in detecting the generated sources, with HOPSfind detecting around $20 \%$ more sources than DUCHAMP. We have used the same cutoff threshold for DUCHAMP and in this 1-deg ${ }^{2}$ data cube, it only detected one spurious source. It would be possible to lower the cutoff and increase the detection rate to more closely match that of HOPSfind, but this introduces more spurious detections that must later be removed. We note that DUCHAMP can also perform an à trous wavelet reconstruction, which determines the amount of structure present and then removes random noise from the cube. This can significantly improve the source detection statistics for DUCHAMP. DUCHAMP, with the à trous wavelet reconstruction incorporated is shown in Figure 4 as the dotted line with crosses. For this implementation, we used three dimensions for reconstruction (i.e. the full cube is reconstructed in one go) and a reconstruction threshold of 2.5. It can be seen that with the à trous wavelet-reconstruction method, DUCHAMP does indeed perform better, with results similar to HOPSfind.

We note that $\mathrm{H}_{2} \mathrm{O}$ masers exhibit a range of line widths, whereas to estimate completeness limits, we have only

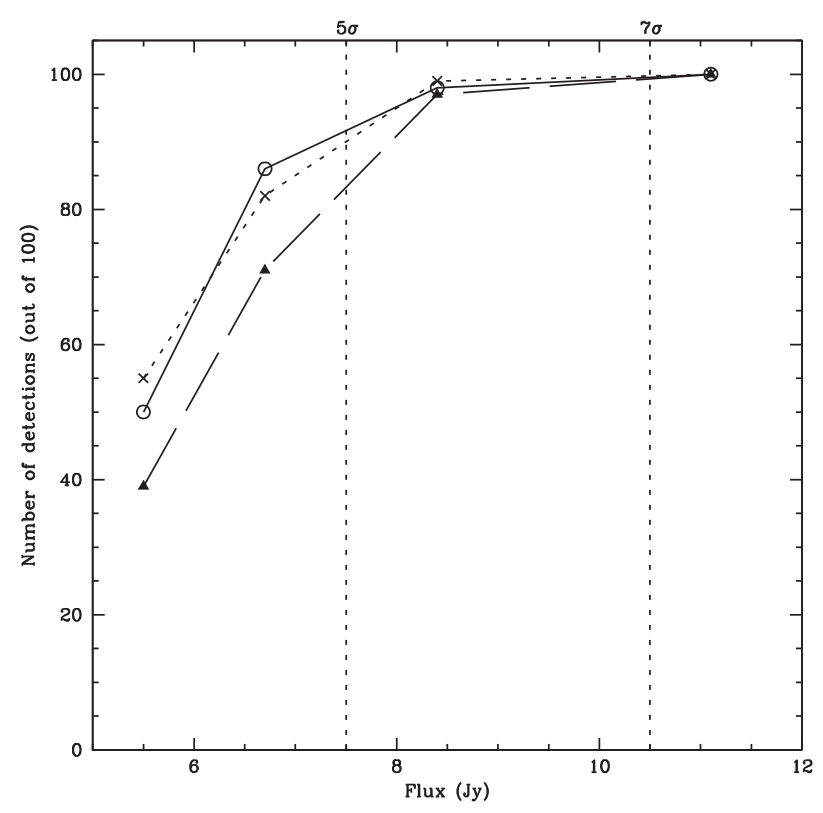

Figure 4 The number of successful detections of generated sources, out of a possible 100, is plotted against the peak flux density of the generated source, using HOPSfind (solid line and open circles), DUCHAMP (dashed line and filled triangles) and DUCHAMP with à trous wavelet reconstruction (dotted line and crosses). The vertical lines represent 5- and 7-times the rms noise level. All methods perform similarly, with the success of detection using DUCHAMP on its own slightly lower than the other two.

injected sources with a single line width. However, we do not expect this will significantly affect our completeness comparison. This is because firstly $85 \%$ of all masers have linewidths within a factor of two from the average $1.4 \mathrm{~km} \mathrm{~s}^{-1}$. Secondly, we expect both HOPSfind and DUCHAMP to perform similarly in detecting either unusually small-FWHM or large-FWHM masers. Thus, whilst the absolute completeness limits may be affected by the linewidth of the maser emission, we do not expect there to be significant differences between the relative performance of HOPSfind and DUCHAMP.

These results generally show that the final results from each source-finding method are very similar. Our intention is not to show that one source-finding method is better than any other, but we imply that the efficiency of any source-finding method is naturally limited by the noise in the data. Thus, well-tuned source-finding methods will produce similar results and the choice of sourcefinding method should be based on the ease of use, as well as other functionality, such as the ability to characterise extended sources.

\section{Data Visualisation}

Cross-matching HOPS datasets with complementary Galactic plane surveys is a key step towards achieving the science goals of the project. For example, matching $\mathrm{NH}_{3}$ clouds and $\mathrm{H}_{2} \mathrm{O}$ masers to features in the mid-infrared Midcourse Space Experiment (Egan \& Price 1996) makes it possible to determine the physical properties of star 
forming regions. To facilitate visualising the data we used the CHROMOSCOPE ${ }^{3}$ software to directly compare largescale HOPS images with other surveys. CHROMOSCOPE is an all-sky image-viewer which allows the user to easily move around the sky, fade between co-registered images and zoom-in to inspect features of interest.

CHROMOSCOPE is JAVASCRIPT-based, so it will run in any modern web-browser and is an ideal tool for publicising images online to the general public. However, it does not require an internet connection and has been used to present data at conferences, and to select interesting objects for follow-up studies. The software supports Keyhole Markup Language (KML) files, used to annotate positions of objects in the popular GOOGLE SKY ${ }^{4}$. Tools are also provided to convert astronomical catalogues to KML format for overplotting on the images.

If an internet connection is available CHROMOSCOPE can utilise the astronomical name resolver ' $\operatorname{lookUP}{ }^{5}$ to find and display specific astronomical objects. The HOPS data is presented on CHROMOSCOPE at http://www. ast. leeds.ac.uk/hops / chromoscope .

\section{Conclusions}

We have compared two methods for finding $\mathrm{H}_{2} \mathrm{O}$-maser sources in the HOPS data. First, we used HOPSfind, which is a manual method of searching for signals in a peak temperature map, that is binned in such a way as to give the greatest contrast to typical $\mathrm{H}_{2} \mathrm{O}$-maser profiles. Second, we used DUCHAMP, which is an automated method of finding masers.

Overall, we find the two methods of detecting sources to perform similarly well. In order to detect weak masers, it is always necessary to also accumulate spurious detections, which must later be assessed through followup observations. We find some minor limitations to the DUCHAMP method because spurious detections that are the result of scanning artifacts are not flagged out. These limitations could be overcome in the future by rejecting candidate detections that do not conform to the beam size and shape or by running DUCHAMP on a signalto-noise cube which has had lower-order polynomials subtracted from the baselines.

The DUCHAMP method finds proportionately more spurious sources than HOPSfind, given the same detection limit. However, when including the à trous wavelet reconstruction method, we find similar detection efficiencies. This is because the detection efficiency is not strongly dependant on the method used, but rather the noise characteristics of the data. Thus, the choice of source-finding method should be based on ease of use and other features, rather than detection efficiency.

We conclude that HOPSfind is more appropriate to detecting $\mathrm{H}_{2} \mathrm{O}$ masers in HOPS, since they are few and far between, as well as being spatially unresolved, so that no extended emission needs to be characterised. For surveys of extended emission, such as the ammonia inversion transitions detected in HOPS (Purcell et al. 2011), DUCHAMP is a better source finding method to use, since it automatically characterises the extended emission. It is expected that in future surveys, such as those planned with ASKAP, many more sources will be detected, making a manual method like HOPSfind impractical. For example, GASKAP expects to find around $15000 \mathrm{OH}$ masers. In addition to this, extended emission from thermal line and continuum emission needs to be characterised, making DUCHAMP the more appropriate source finder.

\section{Acknowledgments}

The Mopra radio telescope is part of the Australia Telescope National Facility which is funded by the Commonwealth of Australia for operation as a National Facility managed by the CSIRO. The authors would like to thank Stuart Lowe from Las Cumbres Observatory Global Telescope Network who wrote CHROMOSCOPE and helped greatly in the creation of the HOPS CHROMOSCOPE website.

\section{References}

Barlow, M. J. et al., 1996, A\&AL, 315, 341

Caswell, J. L. et al., 2010, MNRAS, 404, 1029

Cheung, A. C., Rank, D. M. \& Townes, C. H., 1969, Nature, 221, 626

Claussen, M. J. et al., 1984, ApJL, 285, 79

Claussen, M. J., Wilking, B. A., Benson, P. J., Wootten, A., Myers, P. C. \& Terebey, S., 1996, ApJS, 106, 111

Dickinson, D. F., 1976, ApJS, 30, 259

Egan, M. P. \& Price, S. D., 1996, AJ, 112, 2862

Forster, J. R. \& Caswell, J. L., 1999, A\&AS, 137, 43

Forster, J. R. \& Caswell, J. L., 2000, ApJ, 530, 371

Gundermann, E., 1965, PhD Thesis, Harvard University, Cambridge, MA, USA

Hinkle, K. H. \& Barnes, T. G., 1979, ApJ, 227, 923

Johnston, S. et al., 2007, PASA, 24, 174

Miranda, L. F., G'omez, Y., Anglada G. \& Torrelles, J. M., 2001, Nature, 414, 284

Purcell, C. R. et al., 2011, MNRAS, submitted

Voronkov, M. A., Sobolev, A. M., Ellingsen, S. P. \& Ostrovskii, A. B., 2005, MNRAS, 362, 995

Walsh, A. J., Burton, M. G., Hyland, A. R. \& Robinson, G., 1998, MNRAS, 301, 640

Walsh, A. J., Myers, P. C., Di Francesco, J., Mohanty, S., Bourke, T. L., Gutermuth, R. \& Wilner, D., 2007, ApJ, 655, 958

Walsh, A. J., Lo, N., Burton, M. G., White, G. L., Purcell, C. R., Longmore, S. N., Phillips, C. J. \& Brooks, K. J., 2008, PASA, 25, 105

Walsh, A. J. et al., 2011, MNRAS, 416, 1764

Weaver, H., Williams, D. R. W., Dieter, N. H. \& Lum, W. T., 1965, Nature, 208, 29

Whiting, M., 2011, MNRAS, submitted

Williams, J. P., de Geus, E. J. \& Blitz, L., 1994, ApJ, 428, 693

\footnotetext{
${ }^{3}$ http: / / www. chromoscope. net

https://github.com/slowe/Chromoscope/

${ }^{4}$ http: / / www google.com/sky

${ }^{5}$ http: / /www.strudel.org.uk/lookUP /
} 